\title{
El infrecuente signo de la cruz en epilepsia del lóbulo temporal: reporte de caso documentado con video-EEG.
}

The infrequent sign of the Cross in temporal lobe epilepsy: case report documented with video-EEG.

\begin{abstract}
Elliot Barreto-Acevedo ${ }^{1,2, a}$, Leila Barreto-Barra ${ }^{1, a}$, Mirla Villafuerte-Espinoza ${ }^{1,2, a}$, Alberto Díaz- Vásquez ${ }^{1, a}$, Alicia Becerra-Zegarra ${ }^{3, b}$, Hernández-Vizarreta José ${ }^{1, a}$, Victoria Llaja-Rojas ${ }^{1,4, c}$.
\end{abstract}

\section{RESUMEN}

Las manifestaciones de religiosidad en epilepsia han sido descritas empíricamente desde la antigüedad. En los últimos 60 años con el uso del EEG y luego con la ayuda del Video-EEG se han estudiado mejor estas manifestaciones, en especial en su presentación peri-ictal. El signo de la cruz es un automatismo epiléptico muy infrecuente, con escasos reportes desde el 2009. Presentamos un caso de epilepsia del lóbulo temporal con este automatismo, con estudio detallado que incluyó el registro de la semiología por Video-EEG. Revisamos la literatura con el objetivo de entender los mecanismos fisiopatogénicos propuestos y conocer el valor localizador y/o lateralizador de este signo.

PALABRAS CLAVE: Epilepsia del lóbulo temporal, signo de la cruz, Video-EEG.

\section{SUMMARY}

Religious manifestations in epilepsy have been described empirically since ancestral times. In the last 60 years with the use of the EEG and then with the help of the Video-EEG, these manifestations have been studied better, especially in their peri-ictal presentation. The Sign of the Cross is a very infrequent epileptic automatism, with few reports since 2009. We present a case of temporal lobe epilepsy with this automatism, with a detailed study that included the video-EEG recording of semiology. We review the literature with the objective of understanding the physiopathogenic mechanisms and know the localizing and / or lateralizing value of this sign.

KEY WORDS: Temporal lobe epilepsy, sign of the Cross, Video-EEG.

\section{INTRODUCCIÓN}

La asociación entre religiosidad y epilepsia es antigua. Los griegos y hebreos ya observaban una tendencia para la expresión de manifestaciones religiosas cercana a la ocurrencia de las crisis. A pesar de los intentos de Hipócrates de disociar la epilepsia y la religión; en el siglo XVI y XVII, durante la Cristiandad, se le dio una connotación de posesión demoniaca a la epilepsia. En el siglo XIX, sin embargo, la religiosidad en la epilepsia atrajo el interés científico de neurólogos y psiquiatras en instituciones

\footnotetext{
1 Unidad de Epilepsia, Departamento de Neurología, Hospital Nacional Edgardo Rebagliati Martins, EsSalud. Lima, Perú.

2 Facultad de Medicina, Universidad Nacional Mayor de San Marcos. Lima, Perú.

3 Servicio de Neurocirugía Funcional, Hospital Nacional Edgardo Rebagliati Martins, EsSalud. Lima, Perú.

4 Facultad de Psicología, Universidad Nacional Mayor de San Marcos. Lima, Perú.

a $\quad$ Médico-Neurólogo; ${ }^{\mathrm{b}}$ Médico-Neurocirujano de Epilepsia; ${ }^{\mathrm{c}}$ Neuropsicóloga.
} 
El infrecuente signo de la cruz en epilepsia del lóbulo temporal: reporte de caso documentado con video-EEG.

mentales en Europa (1). Posteriormente se ha realizado un análisis retrospectivo de las manifestaciones de religiosidad en personajes históricos como Sócrates, el Apóstol San Pablo, Buda, Julio Cesar, Mahoma, Dostoievski y Juana de Arco, concluyendo que dichas manifestaciones podrían haberse tratado de crisis epilépticas. Era conocido que algunos de ellos padecían de epilepsia $(2,3)$. Desde el siglo pasado, con el advenimiento del electroencefalograma (EEG) y posteriormente del Video-EEG, se han caracterizado mejor las manifestaciones religiosas en epilepsia; estas manifestaciones han sido reportadas con escasa frecuencia en la literatura $(0,4$ a $4 \%)$ y las siguientes presentaciones semiológicas han sido descritas: Auras de éxtasis o alucinaciones auditivas y visuales de contenido religioso, delusiones y experiencias religiosas en el postictal, hiperreligiosidad interictal, trastorno de personalidad caracterizado por hiperreligiosidad, hipergrafía, alteración de la sexualidad, viscosidad en el pensamiento y disforia, denominado síndrome de Gastaut-Geschwind; rezo o plegaria ictal y automatismos ictales gestuales de rezo como manos en posición de plegaria o postración religiosa (3-8). A pesar de que la mayoría de manifestaciones religiosas asociadas a la epilepsia han sido descritas en pacientes cristianos, también hay reportes en otras religiones como el budismo o incluso en afrodescendientes practicantes de Vooodoo $(4,8)$.

El signo de la cruz es un acto ritual del cristianismo, realizado como parte de un acto litúrgico o devoto, en el cual la mano toca secuencialmente la frente, el esternón y ambos hombros, dibujando una cruz mediante el trazo de 2 líneas que se intersectan en ángulo recto e indican reverencia al madero en el cual Jesucristo fue crucificado. En el Perú, como en algunos otros países latinoamericanos, el cristianismo y en especial el catolicismo es la religión más prevalente. En la crisis epilépticas, especialmente focales con compromiso de conciencia, los automatismos pueden ser variados en su presentación y algunos tienen valor lateralizador y/o focalizador de la zona epileptógena (ZE). En la epilepsia del lóbulo temporal (ELT), son frecuentes los automatismos manuales simples tipo búsqueda y los automatismos orales. El signo de la cruz, es un automatismo manual complejo reportado en la literatura mundial en pocos pacientes desde el 2009 (9-12). Presentamos un caso de epilepsia del lóbulo temporal quien presentó una crisis con este automatismo durante el monitoreo prolongado con video-EEG en nuestra Unidad de Epilepsia y revisamos la literatura mundial al respecto; con los objetivos de conocer si esta manifestación tiene un valor lateralizador $\mathrm{y} / \mathrm{o}$ focalizador $\mathrm{y}$ describir las teorías sobre su fisiopatogenia.

\section{Descripción del caso}

Mujer de 42 años, diestra, católica, secretaria de parroquia desde los 30 años, previo a ello no se consideraba ferviente devota. Su desarrollo psicomotor y escolaridad fueron normales. Tiene epilepsia focal farmacorresistente, con edad de inicio a los 9 años. De los 9 a 16 años escasas crisis focales de parada comportamental de 1 minuto, sin más detalle en el relato. Desde los 32 años, crisis focales con compromiso de conciencia: aura de "sensación de extrañeza" y autosautoscopía; seguidas de parada comportamental con automatismos orales y manuales simples. En algunas crisis repite "tengo que rezar" o "tengo que limpiar". Las crisis son diurnas en vigilia y la frecuencia es de 5/mes. Ha recibido Fenitoína y Carbamazepina sin éxito. Esquema actual: Valproato $2 \mathrm{~g} /$ día y Levetiracetam $3 \mathrm{~g}$ /día. La paciente ingresó

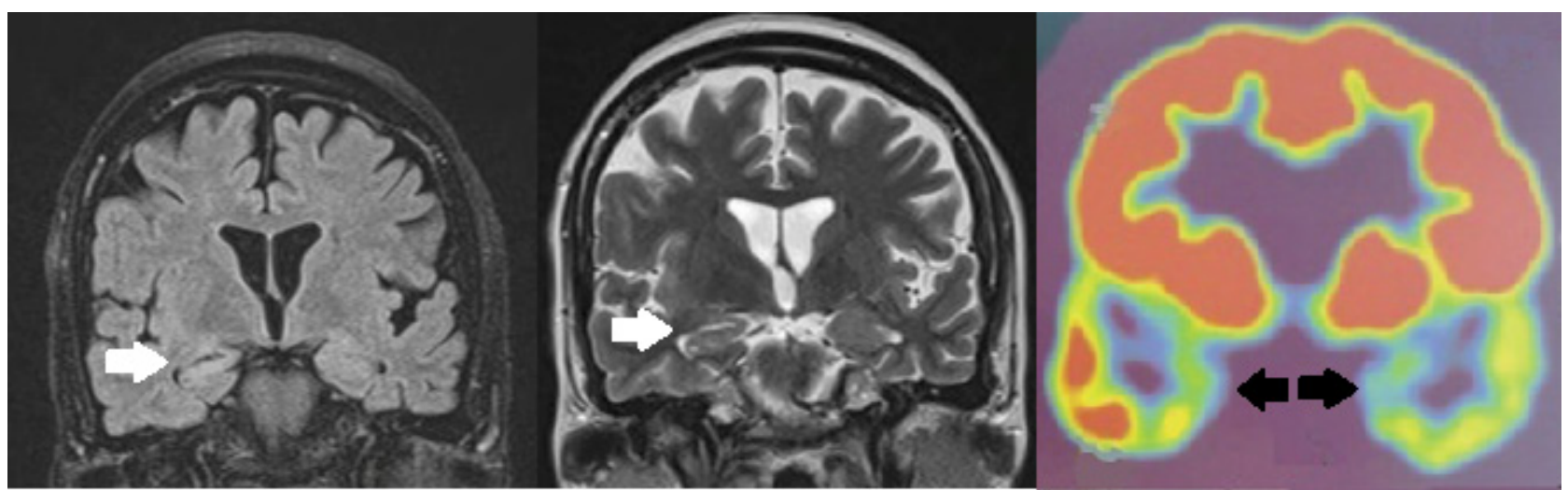

Figura 1. RM de encéfalo (secuencias FLAIR y T2 en cortes coronales) que evidencia esclerosis mesial temporal derecha (flechas blancas) y PET interictal que evidencia hipometabolismo bilateral a predominio izquierdo (flechas negras). 


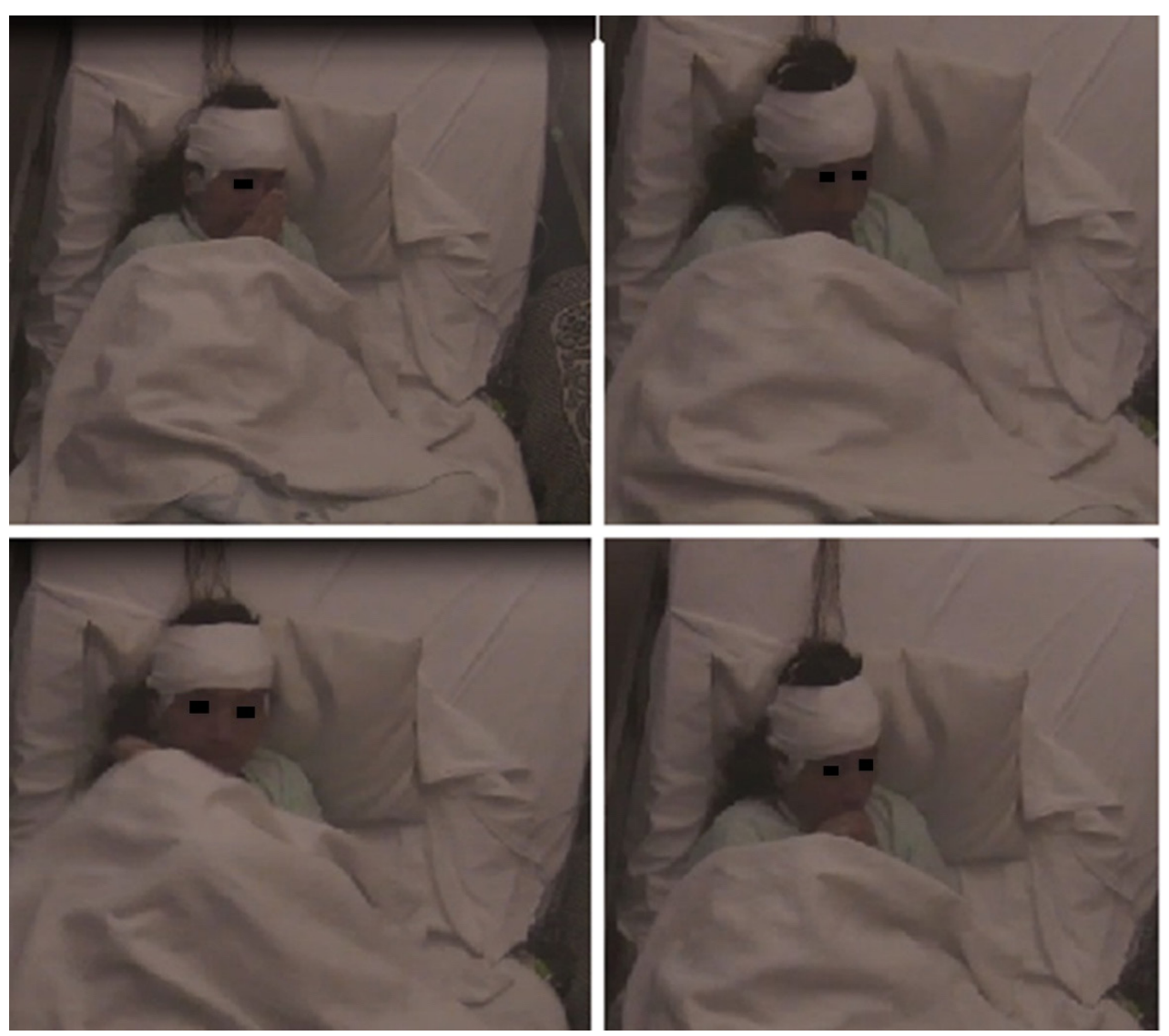

Figura 2. Captura de Video-EEG con el signo de la cruz ictal (automatismo con acto de persignarse).

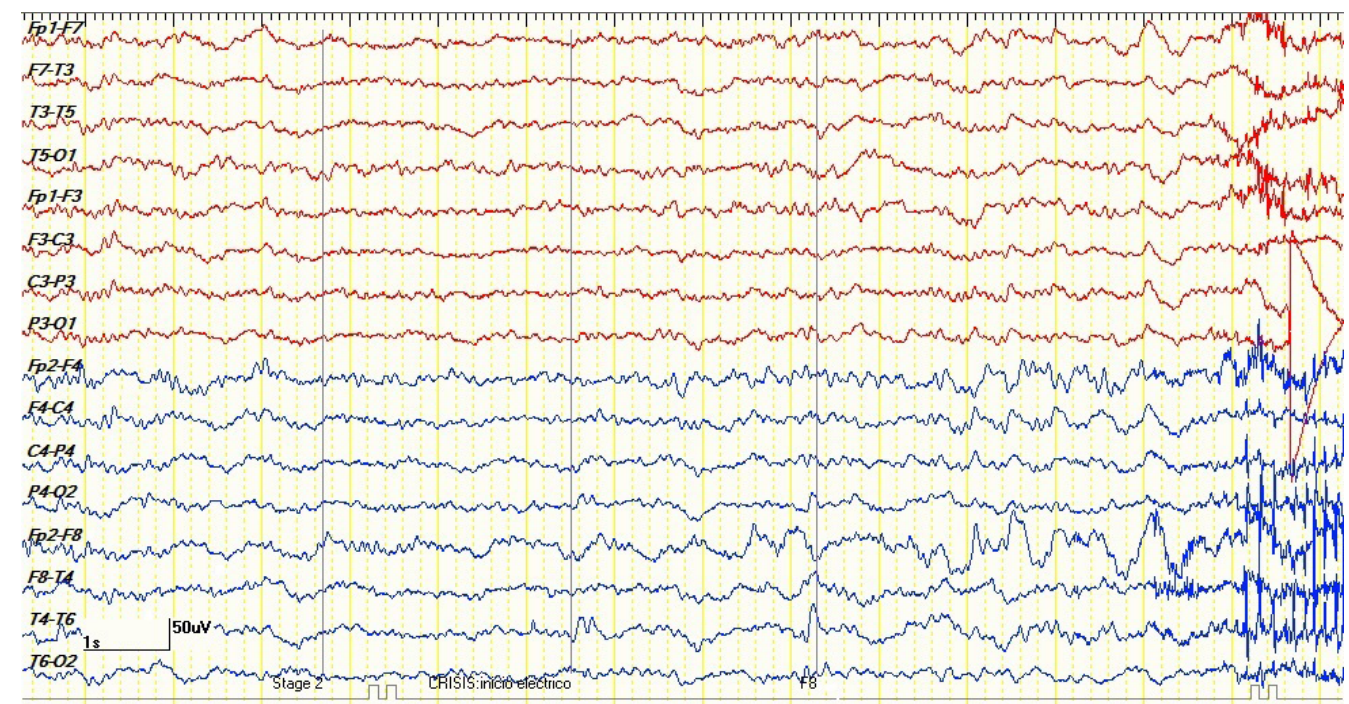

Figura 3. Actividad eléctrica ictal de la crisis focal con compromiso de conciencia y automatismo del signo de la cruz. Inicio eléctrico temporal anterior derecho.

a la Unidad de Epilepsia del Hospital Rebagiati para evaluación prequirúrgica de cirugía de epilepsia. El examen neurológico fue normal, la resonancia (RM) de encéfalo evidenció: Esclerosis mesial temporal derecha (figura 1). La evaluación neuropsicológica evidenció: CI normal y compromiso mnésico verbal y no verbal en $60 \%$. Se realizó un Video-EEG de 173 horas que evidenció: escasa actividad epileptiforme 
El infrecuente signo de la cruz en epilepsia del lóbulo temporal: reporte de caso documentado con video-EEG.

interictal temporal anterior derecha y se registraron 3 crisis; 2 diurnas durante vigilia con semiología similar a la descrita en la anamnesis: aura, seguida de parada comportamental, automatismos orales, automatismos manuales bilaterales y confusión postictal. El EEG ictal evidenció inicio temporal anterior izquierdo. Durante el sueño nocturno (NREM-N2) se registró una tercera crisis que inició con despertar, seguido de parada comportamental con breves automatismos bimanuales y automatismo gestual consistente en acto de persignarse en forma repetitiva por 3 veces con la mano derecha, seguido de automatismos orales prominentes; en el postictal continuó con el sueño (figura 2). El EEG de la tercera crisis evidenció actividad epileptiforme ictal de inicio temporal anterior derecho (figura 3). La tomografía por emisión de positrones (PET) interictal evidenció hipometabolismo bitemporal a predominio izquierdo (figura 1). Se diagnosticó epilepsia del lóbulo temporal mesial bilateral con probables focos independientes y esclerosis mesial temporal. La paciente espera colocación de estimulador del nervio vago.

\section{DISCUSIÓN}

Los automatismos epilépticos se clasifican como perseverativos cuando son la continuación de un acto motor intencional previo a la crisis o de novo cuando surgen con o después del inicio ictal $(5,9)$. Se ha discutido si el signo de la cruz podría preceder al inicio ictal y por tanto ser un acto voluntario como una reacción fisiológica al miedo de que se avecina una crisis. Sin embargo, en todos los casos previamente descritos, así como en nuestro caso, se ha presentado una vez iniciado el compromiso de conciencia; por lo que la posibilidad de ser simplemente una reacción al temor de iniciar una crisis se ha descartado y se considera un automatismo de novo (9).

Se ha planteado que los automatismos son el resultado de la desinhibición o liberación de centros motores subcorticales del control cortical durante la crisis o de la activación de ciertas redes neuronales. Algunos estudios han soportado la hipótesis de que las manifestaciones de religiosidad en epilepsia, serian fenómenos cognitivo-emocionales generados por activación de redes neuronales límbicas $(3,9)$. Besocke et al; por ejemplo, describen una caso con ELT, con crisis focales y automatismos de plegaria ictal, quien fue intervenida de lobectomía anteromesial derecha y luego de lo cual presentaba solo 1 a 2 crisis anuales pero la semiología ya no incluía el rezo ictal (8). Sin embargo, es poco probable que se trate de un fenómeno puramente neurobiológico y es más probable, que estas manifestaciones de religiosidad sean además, influenciadas por factores sociales. De hecho, los 2 reportes con mayor número de pacientes con semiología del signo de la cruz, corresponden a 2 países con arraigo cristiano católico importante: Brasil y Colombia $(9,12)$. Así se explicaría, incluso que algunos casos reportados, quienes aun cuando sean cristianos pero no practicantes estrictos hayan presentado el automatismo del signo de la cruz. También esta hipótesis es avalada por el hecho de que ninguno de los casos reportados a la fecha son ateos $(9,12)$. Esta teoría sostiene una adopción sociocultural de este acto motor como parte de la semiología ictal (9).

Wennberg et al., reportaron un caso muy similar al de nuestra paciente, una mujer de 46 años criada como una estricta formación católica, con epilepsia del lóbulo temporal mesial farmacorresistente, con estudios de ZE que sugerían una epilepsia bitemporal con focos independientes, registraron 3 crisis diurnas habituales con automatismos manuales y orales e inicio ictal temporal anteromesial izquierdo, pero además 2 crisis nocturnas con automatismos del signo de la cruz e inicio ictal anteromesial derecho. Cuando le mostraron a la paciente sus crisis nocturnas, ésta no recordaba haber realizado dicho automatismos, tampoco recordaba haber tenido referencia por los testigos de este tipo de crisis y entonces relató que desde niña su madre le había exigido y forzado por años a persignarse después de la ocurrencia de la crisis. Es así, que los autores se plantean si este automatismo podría ser el resultado de una respuesta o conducta ictal condicionada o aprendida, al menos en algunos pacientes como la que reportan. Por tanto esto estaría a favor de una influencia social en el desarrollo de este automatismo pero a través de un proceso de aprendizaje asociativo condicionado. Es decir, el desarrollo de un automatismo mediante un proceso de aprendizaje psicosocial, en el cual una red neural mesiotemporal epileptógena, con las descargas recurrentes en el tiempo habría reclutado y activado un circuito neural de memoria adyacente (10). Nooraine, reportó un caso que avala aún más la hipótesis de la conducta ictal aprendida. Ellos describen el caso de una mujer hindú, de padres hindúes y casada con un hindú, quien no se consideraba como muy religiosa, inicia una epilepsia del lóbulo temporomesial derecho a los 10 años, con crisis focales con compromiso de conciencia, aura de miedo y automatismos manuales simples y orales; pero a los 23 años es partícipe de un retiro cristiano por una semana y luego de ello incorporó a sus crisis 
el signo de la cruz (11). La diferencia con el caso de Wemberg, es el tiempo de exposición al probable estimulo condicionante, que en este caso fue mucho más corto: 1 semana $(10,11)$. El caso reportado por Dong, sobre otro tipo de automatismo religioso (postración ictal) en un paciente chino con epilepsia del lóbulo temporal derecho también refuerza esta hipótesis, este paciente profesaba el budismo, pero no era practicante, aunque solía rendir esporádicamente homenaje a Buda, realizando la postración sobre sus rodillas y llevando el tronco hacia adelante en festivales tradicionales chinos; luego del inicio de su epilepsia incorporó este acto religioso como automatismo ictal (2). Ambos casos hacen dudar de la hipótesis del condicionamiento puramente cultural, como plantea Lin (9); en especial el caso de Nooraine, pues ocurrió en una sociedad hindú, sin predominio del cristianismo (11). En nuestra paciente, es factible plantear una influencia social y a la vez la posibilidad de la incorporación de este automatismo como una conducta ictal aprendida, pues los testigos no habían reportado previamente este automatismo y la paciente se hizo secretaria parroquial y ferviente devota 21 años después de iniciada su epilepsia.

El reporte de Arango-Jaramillo et al, cuenta con el mayor número de pacientes respecto al signo de la cruz y ha tratado de establecer con las limitaciones del caso, por el bajo número de pacientes, el valor lateralizador de este signo (12). Previo a este reporte solo se habían descrito un total de 6 pacientes en 3 reportes en la literatura mundial y el automatismo en cuestión había ocurrido solo en pacientes con epilepsia del lóbulo temporomesial derecho (9-11). Arango-Jaramillo encontraron una prevalencia de $1,1 \%$ de este automatismo en un total de 1308 casos de un centro terciario con programa de cirugía de epilepsia. Nosotros encontramos 1 solo caso en 88 pacientes estudiados con Video-EEG prolongado hasta la fecha en nuestro centro. Ellos encontraron un bajo valor predictivo positivo y negativo como signo lateralizador. De sus 14 casos identificados con este automatismo, solo 11 ocurrieron dentro de la fase ictal; estos 11 pacientes tuvieron epilepsia del lóbulo temporal (6 derechos y 5 izquierdos) (12).

En todos los casos reportados, así como el nuestro; este automatismo se ha realizado con la mano derecha; aun incluso, en los pocos casos con ZE mesial temporal izquierda de la serie de Arango-Jaramillo. Se ha tratado de explicar este hecho por la dominancia hemisférica izquierda en todos los pacientes reportados o por la distonía contralateral en algunos pacientes o que este hecho avalaría que se trate de un fenómeno ictal aprendido. Así mismo este hallazgo refuerza lo referido por Arango-Jaramillo, que sería un signo con pobre valor lateralizador, pues no sería siempre ipsilateral o contralateral a la ZE (9-12).

En conclusión, el automatismo ictal del signo de la cruz es una manifestación epiléptica infrecuente, que ha sido descrito previo a nuestro reporte solo en 17 casos del lóbulo temporal en la literatura mundial; sin embargo por su inusual presentación y el hallazgo de otros reportes tiene pobre valor lateralizador. Sus mecanismos fisiopatogénicos no están del todo esclarecidos; pero tanto factores psicosociales como neurobiológicos pueden estar involucrados.

Los autores no han recibido financiamiento de ninguna institución. El trabajo ha sido autofinanciado. Los autores no tienen conflicto de interés.

\section{Correspondencia}

Dr. Elliot Barreto Acevedo Hospital Nacional Edgardo Rebagliati Martins

Av. Rebagliati 490 Jesús María

Teléfono: 511-265-4901 anexo: 3081. Celular: 989963057.

Correo electrónico: elliotba@hotmail.com

\section{REFERENCIAS BIBLIOGRÁFICAS}

1. McCrae N, Whitley R. Exaltation in temporal lobe epilepsy: Neuropsychiatric symptom or Portal to the Divine? J Med Humanit. 2014; 35:241-55. doi: 10.1007/s10912-014-9294-4

2. Muhammed L. A retrospective diagnosis of epilepsy in three historical figures: St Paul, Joan of Arc and Socrates. J Med Biogr. 2013; 21: 208-11.

3. Devinsky O, Lai G. Spirituality and religion in epilepsy. Epilepsy Behav. 2008; 12: 636-43.

4. Dong L, Zhou X. An uncommon automatism with religious connotation- prostration in a case of right temporal lobe epilepsy. Seizure. 2016; 35: 33-5.

5. Özkara C, Sarý H, Hanoglu L, Yeni N. Aydogdu I, Özyurt E. Ictal kissing and religious speech in a patient with right temporal lobe epilepsy. Epileptic Disord.2004; 6: 241-6

6. Asheim-Hansen B, Brodtkorb E. Partial epilepsy with “ecstatic" seizures. Epilepsy Behav. 2003; 4: 667-73.

7. Carrazana E, De Toledo J, Tatum W, Rivas-Vasquez R, Rey G, Wheeler S. Epilepsy and religious experiences: Voodoo possession. Epilepsia. 1999; 40(2):239-241.

8. Besocke A, Baccanelli M, Cristiano E, García MC, 
El infrecuente signo de la cruz en epilepsia del lóbulo temporal: reporte de caso documentado con video-EEG.

Silva W, Valiensi S. Manifestaciones religiosas como semiología ictal en la epilepsia del lóbulo temporal. Rev Neurol. 2012; 54 (1): 61-3.

9. Lin K, Marx C, Caboclo L, Centeno R, Sakamoto A, Yacubian E. Sign of the cross (signum crucis): observation of an uncommon ictal manifestation of mesial temporal lobe epilepsy. Epilepsy Behav. 2009; 14: 400-3.

10. Wennberg R, McAndrews M, Zumsteg D, Velazquez $\mathrm{J}$. The sign of the cross as a learned ictal automatism? Epilepsy Behav. 2009; 15: 394-8.
11. Nooraine J, Jayaraman A, Reddy S, Iyer R, Raghavendra S. Ictal sign of cross-does it have any religious annotations at all? Seizure. 2013; 22: 584-5.

12. Arango-Jaramillo E, Lozano-García L, BenjumeaCuartas V, Andrade-Machado R. Periictal sign of the cross or Signum Crucis as a lateralizing sign in focal epilepsies: Not only a right temporal lobe epilepsy feature. Epilepsy Behav. 2018; 78: 52-6.

Recibido: 20/11/2018

Aceptado: 10/12/2018 